\title{
Isolated complete bladder neck transaction without pelvic fracture in a child: a rare presentation
}

\author{
Arora S. ${ }^{1}$, Parashar S. ${ }^{2}$, Datey S. ${ }^{3}$, Sharma R. ${ }^{4}$, Lunawat A. ${ }^{5}$ \\ ${ }^{1}$ Dr. Shweta Arora, ${ }^{2}$ Dr. Sanjay Parashar, ${ }^{3}$ Dr. Sanjay Datey, ${ }^{4}$ Dr. Rajesh Sharma, ${ }^{5}$ Dr. Ajay Lunawat, ${ }^{1,3,4,5}$ authors are \\ attached with Department of General Surgery, ${ }^{2}$ author is attached with Department of Urology, Sri Aurobindo Medical \\ College /MPMSU, Jabalpur, MP, India.
}

Corresponding Author: Dr. Shweta Arora, Department of General Surgery, Sri Aurobindo Medical College/MPSMU, Jabalpur, India. E-mail: drshwetasurgery@gmail.com

\begin{abstract}
Background: Urethral injuries account for 3.4\% of the children admitted with traumatic injuries of the genitourinary tracts. Most of the urethral injuries are usually associated with bony injury. Very few of such cases reported without any association with bonetraum. Here, we report a rare case of isolated bladder neck injury without pelvic fracture. Case report: The patient was a 12-year old boy admitted with a history of road traffic accident. Ultrasonography was suggestive of mild free fluid in perivesical region, cystogram was performed and an extravasation near the bladder neck was detected. CT urography revealedinjury at bladder neck and prostate region with contrast extravasation. On exploratory laparotomy there was evidence of Complete transaction of bladder neck present. Primary Vesicourethral end to end anastomosis was done. The post-operative course and follow-up was uneventful as patient had satisfactory urine flow rate with continence intact. Conclusion: The present study highlights our experience with early repair of bladder neck trauma in a child with excellent outcome.
\end{abstract}

Keywords: Complete bladder neck transaction, Children, Pelvic injury

\section{Introduction}

About $80 \%$ of bladder injuries are associated with pelvic fracture but bladder neck injury without pelvic fracture is rare [1]. We describe an interesting case of complete bladder neck transaction without any associated urethral or pelvic injury in a child which was managed surgically in early post trauma period.

Case Report: We report our experience of paediatric patient aged 12 years, who presented with post traumatic bladder neck distraction injurywith history of road traffic accident by fall from motorbike by getting hit with four-wheeler (deceleration injury). Patient came to us 12 hrs post trauma. He was catheterized at some other hospital due to inability to void post injury with mild haematuria in urine bag. He also had right tibio-fibular fracture; there was no other major concomitant injuries. Patient was hemodynamically stable his routine blood investigations are normal and local examination findings and per rectal examination were also normal, he underwent usg abdomen which was s/o mild free fluid in perivesical region.

Manuscript Received: $10^{\text {th }}$ March 2019

Reviewed: $20^{\text {th }}$ March 2019

Author Corrected: $25^{\text {th }}$ March 2019

Accepted for Publication: $29^{\text {th }}$ March 2019
Inretrograde cystography, S/o extra vasation of contrast around bladder neck region. Contrast Enhanced Computed Tomography (CECT) showing injury at bladder neck and prostate region with urine extra vasation, Foley's bulb is seen inside urinoma. For bladder neck trauma, decision for operative intervention was taken.

First urethroscopy was done- Bladder neck could not be visualized, Guide wire placed perurethrally. Exploratory laparotomy was done with lower midline vertical incision. Operative finding was complete disruption of the posterior urethra from the bladder at the level of bladder neck. Primary vesicourethral anastomosis was done over14 numberFoley's catheters with 4-0 vicryl suture in onelayer interrupted fashion. No leak on retrograde filling, $20 \mathrm{~F}$ peri-vesical drain kept.Drain removed on post-operativeday2, sutures removed on post-operative day-10, foleys removed on post-operative day-21 after normal cystography finding.On Voiding trial patient had good urinary flow. In further follow-up patient maintained this flow and remain continent till follow up of 2 years. 


\section{Case Report}

\section{Discussion}

Trauma due to RTA, high velocity falls onto perineum account for majority of childhood urethral injuries. Most of the bladder injuries are associated with pelvic fractures (usually anterior pelvic arch fracture) but bladder neck injury without pelvic fracture is rare. Posterior urethral injuries happen after severe trauma are usually associated with pelvic fractures in 1.5 to $10 \%$ cases. In younger children prostate is soft and small hence fixation with surrounding is lax leading to little stabilization of posterior urethra this makes urethra vulnerable to injury particularly at bladder neck. Rupture of posterior urethra is caused by shearing forces commonly occurring at the apex of the prostate. Primary injury involves prostate and prostatic urethra. Bladder neck gets involved secondarily by extension. Severe displacement of the prostate off the pelvic floor makes a complete posterior urethral disruption more common.

Bladder neck injuries are usually longitudinal in adults while in children transverse bladder neck injuries are common [1]. In our case, it is transverse complete bladder neck transection injury with lateral pelvic force might bea offending cause for such disruption. The classic picture of blood at urethral meatus, inability to void, haematuria on catheterisation perineal ecchymosis or "butterfly hematoma", upward displacement of prostate on digital rectal examination is suggestive of post urethral trauma rectal examination should be performed also to exclude an associated rectal injury.The recommended radiographic method isRUG, an elevated contrast filled bladder with accumulating hematoma and/or urinoma is highly suggestive of bladder neck disruption coincides with findings of our case. Our patient has Type 4 urethral injury according to Goldman Modification of Colapinto and Mc Callum classification of Urethral Injury and type 5 urethral injury on AAST (American association of surgical trauma).

Management: As children with urethral trauma are unstable due to other associated injuries, the first step in management is to stabilize and provide urinary drainage. The outcome objective in such types of injuries is maintenance of continence, potency and limiting possibility of urethral stricture.Immediate management of posterior urethral injuries remains controversial and has three different approaches.

Traditional management of pelvic fracture urethral injury (PFUI) is placement of a suprapubic tube (SPT) and delayed urethroplasty to reconnect the ruptured urethra another approach is Primary endoscopic re-alignment for hemodynamicaly stable patients or advancing a urinary catheter across the posterior urethral ruptures and third approach is primary surgical repair over catheter in early period [1].

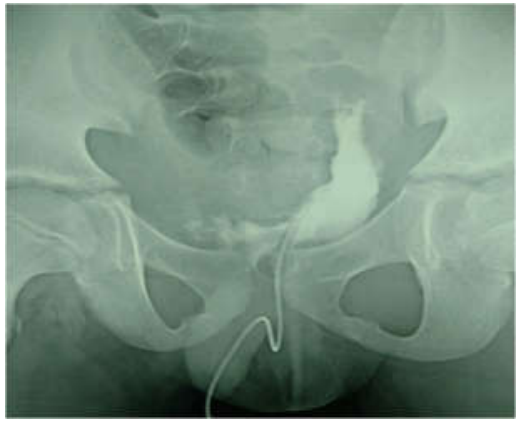

Fig-1: RGU S/O Urinary Extravasation

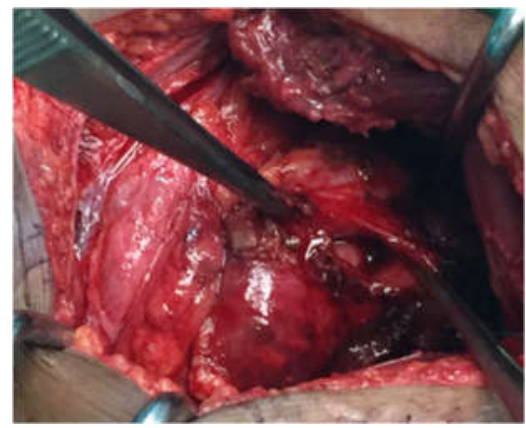

Fig-3: Bladder Neck Rupture

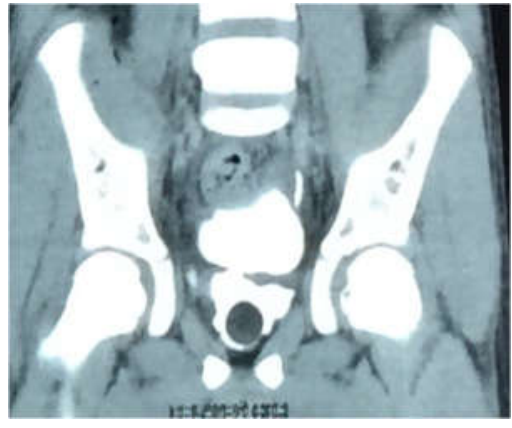

Fig-2: CECT S/O Urinoma around Bladder Neck

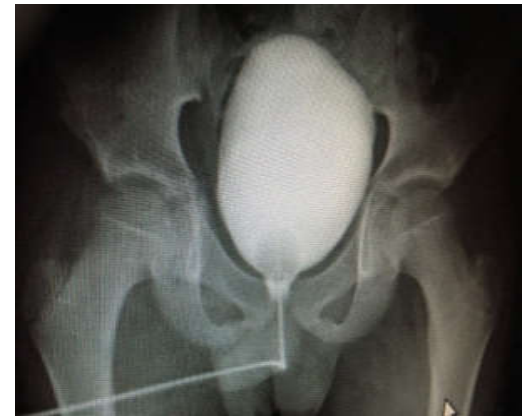

Fig-4: Post Operative Normal Cystogram 


\section{Case Report}

In comparison to adults supra pubic catheterization is not recommended in children because of risk of future incontinence and impotence which is more common when it is performed [2]. Bladder neck and prostatic urethral injuries need to be identified and treated promptly as they do not heal spontaneously and can cause considerable morbidity due to persistent extravasation of urine, pelvic osteomyelitis and permanent incontinence.

\section{AAST Bladder Injury Scale}

\begin{tabular}{ll}
\hline Grade & Description \\
\hline I & $\begin{array}{l}\text { Hematoma: Contusion, intramural hematoma, Laceration: Partial } \\
\text { Thickness }\end{array}$ \\
2 & $\begin{array}{l}\text { Laceration: Extraperitoneal bladder wall laceration }<2 \mathrm{~cm} \\
3\end{array}$ \\
& $\begin{array}{l}\text { Laceration: Extraperitoneal }(>2 \mathrm{~cm}) \text { or intra-peritoneal }(<2 \mathrm{~cm}) \\
\text { bladder wall laceration }\end{array}$ \\
4 & $\begin{array}{l}\text { Laceration: Intraperitoneal bladder wall laceration }>2 \mathrm{~cm} \\
5\end{array}$ \\
\hline
\end{tabular}

Table-1: AAST Grade of Bladder Trauma

\section{Goldman colapinto classification of urethral injuries}

- Type I: stretching of posterior urethra due to disruption of puboprostatic ligaments, though the urethra is intact

- Type II: posterior urethral injury above urogenital diaphragm

- Type III: injury to membranous urethra, extending into proximal bulbous urethra (i.e. with laceration of urogenital diaphragm)

- Type IV: bladder base injury involving bladder neck extending into the proximal urethra

- internal sphincter is injured, hence potential for incontinence

- Type IVa: bladder base injury, not involving bladder neck (cannot be differentiated from type IV radiologically)

- Type V: anterior urethral injury (isolated)

Table-2: Classification of Urethral Injury

The goal of primary realignment is to align both ends of the disrupted urethra so that they heal in the correct position as the pelvic hematoma is reabsorbed [3]. success of primary realignment, is not very encouraging as most eventually require repeated instrumentation and /or formal urethroplasty to maintain patency as post procedure chances of urethral narrowing ranges from $14 \%-100 \%$ in pediatric patients Primary surgical repair, urethral catheter drainage without suprapubic cystostomy is recommended [2].

Follow up : Guidelines recommends patient should be monitored for complications like stricture formation, erectile dysfunction, incontinence for at least one year following urethral injury with uroflowmetry, RUG, cystoscopy. Our patient had good urinary flow with intact continence in follow up period of 1 year. 


\section{Case Report}

\section{Conclusion}

This case shows that even in absence of pelvic fracture and in spite of good urine output, with no significant haematuria, significant bladder neck injury can occur and should be suspected in trauma patients.

Such injuries need early identification and prompt treatment.

Early surgical repair in wisely selected cases is associated with favourable outcome.

Conflict of interest: None declared. Funding: Nil, Permission from IRB: Yes

\section{References}

1. Sawant AS, Kapadnis LA, Kumar V, et al. Paediatric Post-Traumatic Bladder Neck Distraction Injury: Case Series. J Clin Diagn Res. 2017 Feb;11(2):PR03-PR04. doi: 10.7860/JCDR/2017/20782.9244. Epub 2017Feb 1.

2.Cetin Ali Karadag, BurakTander. Laparoscopic repair in children with traumatic bladder perforation J Minim Access Surg. 2016 Jul-Sep; 12(3): 292-294.

3. Pichler R, Fritsch H, Skradski V, et al. Diagnosis and management of pediatric urethral injuries. Urol Int. 2012; 89 (2):136-42.doi:10.1159/000336291.Epub 2012 Mar 19.

\section{How to cite this article?}

Arora S, Parashar S, Datey S, Sharma R, Lunawat A. Isolated complete bladder neck transaction without pelvic fracture in a child: a rare presentation. Surgical Update: Int J surg Orthopedics. 2019;5(2):70-73.doi:10.17511/ijoso.2019.i02.01. 